Published in final edited form as:

Nat Neurosci. 2005 January ; 8(1): 72-78.

\title{
High frequency, synchronized bursting drives eye-specific segregation of retinogeniculate projections
}

\author{
Christine L. Torborg, Kristi A. Hansen, and Marla B. Feller \\ Neurobiology Section, Division of Biological Sciences, UCSD, La Jolla, CA, USA.
}

\section{Abstract}

Blockade of retinal waves prevents the segregation of retinogeniculate afferents into eye-specific layers in the visual thalamus. However, the key features of retinal waves that drive this refinement are controversial. Some manipulations of retinal waves lead to normal eye-specific segregation but others do not. By comparing retinal spiking patterns in several transgenic mice with differing levels of eye-specific segregation, we show that the presence of high frequency bursts synchronized across neighboring retinal ganglion cells correlates with robust eye-specific segregation, and that the presence of high levels of asynchronous spikes does not inhibit this segregation. These findings provide a possible resolution for previously described discrepancies regarding the role of retinal waves in retinogeniculate segregation.

Spontaneous retinal activity is required for the formation of eye-specific layers in the dorsal lateral geniculate nucleus of the thalamus (dLGN) from an initially overlapping distribution of retinogeniculate axons ${ }^{1-5}$. In the mouse, initial segregation of retinogeniculate afferents into eye-specific layers occurs during the first postnatal week and consists of the coalescence of ipsilaterally-projecting axons in the dorsomedial region of the $\mathrm{dLGN}^{6}$. Eye-specific segregation continues during the second postnatal week to refine the boundaries of the ipsilateral layer ${ }^{7}$ or in experimental animals in which a defined ipsilateral layer does not develop, to drive local segregation in the absence of layers ${ }^{7-9}$. It is hypothesized that presynaptic activity patterns contain the information for sorting retinal ganglion cell axons into eye-specific regions $10-12$. Retinal waves drive individual retinal ganglion cells (RGCs) to fire short bursts of action potentials that are correlated amongst nearby cells followed by long periods of silence. This pattern of firing ensures that 1) nearby RGCs that converge onto the same LGN neuron fire together in bursts, providing sufficient excitation to depolarize the postsynaptic cell above threshold ${ }^{13}$, and 2) neighboring RGCs are not likely to fire at the same time as retinotopically-matched RGCs from the other retina. However, the essential features of spontaneous retinal activity and the plasticity rules that drive activity-dependent eye-specific segregation are not yet known.

One approach to determine the essential features of spontaneous retinal activity for eye-specific segregation is to identify genetically-altered mice that have disrupted activity patterns with maintained retinal ganglion cell firing. Previously we have shown that mice lacking the $\beta 2$ subunit of the nAChR ( $\beta 2-/$ - mice) do not have retinal waves but exhibit sustained firing of individual RGCs ${ }^{14}$ and as a result do not segregate into layers ${ }^{7}$. In contrast, other manipulations that disrupt firing patterns do not alter eye-specific segregation and layer formation 4,5 .

Correspondence should be addressed to Marla B. Feller, Neurobiology Section, 0357, UCSD, 9500, Gilman Dr. La Jolla, CA, 92093-0357, mfeller@ucsd.edu. 
In this study we characterized retinal firing patterns and retinogeniculate segregation in mice lacking the gap junction protein, Connexin 36 (Cx36). We found that although Cx36-/- mice have significantly disrupted correlations during the second postnatal week, eye-specific segregation proceeded normally. We also found that in $\beta 2-/-\mathrm{Cx} 36-/-$ mice, which never have normal spontaneous firing patterns during the period of eye-specific segregation, RGC axons segregate into local eye-specific regions even in the absence of layers. By comparing the spike trains among WT, Cx36-/- mice, $\beta 2-/-$ mice and $\beta 2-/-\mathrm{C} \times 36-/-$ mice, we were able to identify several features of retinal firing patterns that appear to be necessary for driving eye-specific segregation.

\section{RESULTS}

\section{Multi-electrode array recordings demonstrate Cx36-/- retinas have disrupted retinal firing patterns}

Pharmacological studies suggest that gap junctions may be involved in retinal wave propagation 15 . Only two connexin types, connexin 36 and connexin 45 , have been localized unambiguously to the inner mammalian retina 16,17 , implicating one or both of these connexins in correlating RGC firing during development. We examined genetically altered mice in which the gene for $\mathrm{Cx} 36$ has been substituted by the reporter LacZ ${ }^{18}$. Histochemistry revealed the onset of $\beta$-galactosidase ( $\beta$-gal) expression in the inner retina at postnatal day 2 (P2) as discrete puncta in the ganglion cell layer. $\beta$-gal expression in the ganglion cell layer and inner nuclear layer gradually increased until the adult pattern of expression emerged 19 (Figure 1), and therefore may play a role in generating retinal waves.

Using a multielectrode array, extracellular recordings made from P4 and P5 WT retinas revealed that most RGCs fire bursts of action potentials followed by long periods of silence (Figure 2A14), similar to the firing patterns seen in neonatal ferrets 20 . These bursts were correlated among nearby RGCs as propagating retinal waves (Supplemental Movie 1). At P4-5, most Cx36-/- RGCs had firing patterns qualitatively similar to WT (Figure 2A, Supplemental Movie 2), with the exception of $15 \%$ of $\mathrm{Cx} 36-/$ - retinal neurons that fired tonically at low firing rates between bursts (Figure 2A, bottom spike train). This suggests that the absence of Cx36 at this age may contribute to the excitability of a subset of retinal neurons.

Firing patterns of Cx36 -/- retinal neurons were dramatically altered in the second postnatal week. Multi-electrode array recordings at P10-11 demonstrated that WT retinal neurons still exhibited highly correlated bursts separated by long silent periods 14,21 . However, in Cx36-/mice, many retinal neurons remained active throughout the recording (Figure 2B). The level and type of activity varied widely across retinal neurons, from few spikes in between bursts to nearly tonic firing. Though the P10-11 Cx36-/- mice still exhibited propagating retinal activity (Supplemental Movie 3), the additional sustained firing increased the probability that RGCs from two eyes were firing simultaneously, potentially inhibiting eye-specific segregation.

\section{Cx36-/- mice have normal eye-specific segregation}

To test directly whether altered patterns of activity could drive eye-specific segregation, we compared retinogeniculate projections between WT and Cx36-/- mice ${ }^{7,22}$. By P8 in both WT and $\mathrm{Cx} 36-/-$ mice, the ipsilateral axon terminal fields have coalesced to form a layer that lacks contralateral axon terminals (Figure 3A, left). Based on two independent methods of quantification 22 , the extent of eye-specific segregation of Cx36-/- retinogeniculate axons at both P8 and P14 was indistinguishable from WT (Figure 3A, column 4). In addition, segregation in Cx36-/- mice increased significantly between P8 and P14, similar to WT (Figure 3B). These findings indicate that the altered firing detected in $\mathrm{Cx} 36-/$ - retina drives normal eye-specific segregation of retinogeniculate afferents. 


\section{Reduced retinal correlations can drive eye-specific segregation from an unlayered pattern}

One potential explanation for the observation that P14 Cx36-/- mice have normal eye-specific segregation is that a substantial amount of segregation has already occurred by the time activity is maximally disrupted at P10. To demonstrate that the disrupted activity is sufficient to drive segregation from an initially unlayered pattern, we crossed the Cx36-/- with $\beta 2-/-$ mice. The latter have uncorrelated retinal activity during the first postnatal week 14,23 and no segregation into eye-specific layers by P8 3,7 . We expected $\beta 2 /-\mathrm{Cx} 36-/$ - mice to have no layers at the end of the first week and disrupted activity throughout the first and second week, and therefore we used them to test whether disrupted activity can drive eye-specific segregation from an initially unsegregated pattern.

Multielectrode array recordings from P8 and P10 $\beta 2-/-C x 36-/$ - retinas revealed disrupted retinal activity. These retinas had both bursting and tonically firing neurons (Figure 4A), though there were not as many tonically firing cells as in Cx36-/- retinas. By P8, the ipsilateral projection of $\beta 2-/-\mathrm{Cx} 36-/-$ mice was not confined to the dorsomedial region and retinogeniculate afferents from the two eyes did not segregate (Figure 4A). This phenotype is identical to that observed in P8 $\beta 2-/-$ mice 3,7 . In P14 $\beta 2-/-C \times 36-/-$ mice, a patchy distribution of ipsilateral projections emerged (Figure 4B, left), and there was a significant increase in eyespecific refinement of retinogeniculate afferents from that observed at P8 (Figure 4C and D). This segregation pattern is similar to that observed in P14 $\beta 2-/-$ mice 7 , in which waves reemerge during the second postnatal week ${ }^{14,23}$. Thus, we conclude that both normal spiking patterns in P10-11 $\beta 2-/-$ mice and disrupted retinal spiking patterns in P10-11 $\beta 2-/-\mathrm{Cx} 36-/-$ mice drive local eye-specific segregation from an unlayered retinogeniculate projection pattern.

\section{Correlated high frequency bursts may be essential features for eye-specific segregation}

We have established that the firing patterns in WT, Cx36-/-, P10-11 $\beta 2-/-$, and P10-11 $\beta 2-/-$ $\mathrm{Cx} 36-/$ - mice drive eye-specific segregation of retinogeniculate afferents while altered firing patterns in P4-P5 $\beta 2-/$ - mice cannot. One hypothesis is that the altered Cx36-/- firing patterns retain features of WT firing patterns that are absent from the firing patterns of P4-5 $\beta 2-/$ - mice. To identify the features that are essential for eye-specific segregation of RGC afferents, we compared several properties of the spiking patterns across ages and genotypes.

First, we compared the firing properties of individual cells. The overall level of activity (the total number of spikes per minute) was similar across all genotypes and ages except in P10-11 Cx36-/- mice, where it was significantly increased relative to WT and $\beta 2-/$ - mice (Figure 5A). The instantaneous firing rate is decreased relative to WT in both P4-5 $\beta 2-/-$ and P10-11 Cx36-/retinas (Figure 5B). The percentage of time individual retinal neurons fire at greater than $1 \mathrm{~Hz}$ was increased in both P10-11 Cx36-/- and P4-5 $\beta 2-/$ - mice relative to WT mice (Figure 5C). These three features did not distinguish WT and Cx36-/- retinas from $\beta 2-/$ - retinas; therefore, they are not essential features for driving eye-specific segregation.

In contrast, the percentage of time retinal neurons spent firing at greater than $10 \mathrm{~Hz}$ was significantly higher for WT and Cx36-/- retinal neurons than P4-5 $\beta 2$ retinal neurons (Figure 5D). Since these bursts occurred infrequently (Figure 5D), it was not likely that retinotopically matched neurons in the two eyes fired bursts simultaneously. Hence, bursts of high frequency action potentials may be an essential feature for driving eye-specific segregation.

To compare the correlations across cells within an eye, we computed the correlation index as a function of distance between cells for all genotypes $14,24,25$. In WT retinas, the correlation index was highest for nearby neurons and fell off as a function of distance between cell pairs (Figure 6A). As reported earlier, P4-5 $\beta 2$ mice had dramatically reduced nearest neighbor 
correlation ${ }^{14}$. Similarly, P10-11 Cx36-/- mice had dramatically reduced correlation indices at all distances (Figure 6B) but in contrast to $\beta 2$ mice, nearby cell pairs had a larger correlation index than distant cell pairs (Figure 6B, dotted line). This result was not dependent on the timescale of the correlation since varying the time window from $\pm 10 \mathrm{~ms}$ to $\pm 500 \mathrm{~ms}$ did not change the correlation index significantly (Supplemental Figure 1). Hence, we conclude that a second essential feature for driving eye-specific segregation may be that nearby cells fire in a more correlated fashion than distant cells, independent of the magnitude of the correlation.

Since the correlation index was normalized by the number of spikes in both cells, the reduction in the magnitude of the correlation index in P10-11 Cx36-/- mice was due in part to overall increase in the total number of spikes (Figure 4A). Therefore, as a second measure of spatial correlations, we calculated the absolute number of correlated spikes across genotypes. To calculate the number of correlated spikes, we generated a third spike train that included only those spikes in cell A that were synchronous to within $\pm 100 \mathrm{~ms}$ with a spike in cell B and vice versa. Since cells in WT mice had very few asynchronous spikes, the newly generated spike trains were very similar in pattern to the original spike train (Figure 6C, top). In P10-11 Cx36-/mice, asynchronous spikes were not retained and the firing patterns are similar to the bursts detected in WT mice (Figure 6C, middle). In P4-5 $\beta 2-/$ - retinas, since most of the spikes were asynchronous, the newly generated spike trains contained very few spikes (Figure 6A, bottom). The number of synchronized spikes between two neighboring neurons in Cx36-/- and WT mice was significantly higher than $\beta 2-/$ - mice (Figure $6 \mathrm{D}$ ), and may also be an essential feature for driving eye-specific segregation.

Previously, it has been demonstrated that intraocular injection of adenylate cyclase activators in ferret modifies retinal wave activity but does not alter the extent of eye-specific segregation ${ }^{4}$. We tested whether the retinal firing patterns in the presence of the adenylate cyclase activator, forskolin, maintained the essential features for driving eye-specific segregation, which were the percentage of time individual RGCs fired above $10 \mathrm{~Hz}$ and the number of synchronized spikes, as identified in Figures 5 and 6. As previously reported ${ }^{4,26}$, the presence of forskolin greatly increased the frequency of bursts (Figure 7A). There was an overall increase in the number of action potentials fired, but not in the median firing rate within a burst. Importantly, there was an increase in the percentage of time individual RGCs fired above $10 \mathrm{~Hz}$ and in the number of synchronized spikes (Figure 7B). Hence, bath application of forskolin did not decrease the correlated firing patterns nor the percentage of time firing above $10 \mathrm{~Hz}$, in contrast with the $\beta 2-/$ - mouse, which reduced both parameters. These findings are consistent the conclusion that a threshold level of bursting and nearest neighbor correlations are required for driving eye-specific segregation.

\section{DISCUSSION}

In summary, Cx36-/- mice exhibit significantly disrupted retinal firing patterns during the second postnatal week (Figure 2) but normal refinement of eye-specific segregation (Figure 3). Similarly, double mutant $\beta 2-/-C x 36-/-$ mice, which never have normal retinal activity during the first two weeks, have substantial refinement into local eye-specific regions. By analyzing various properties of the spikes trains, we found that these altered activity patterns retained key features of the pattern in WT mice: individual cells still fired in bursts at greater than 10 $\mathrm{Hz}$ (Figure 5D), and the firing of nearest neighbors remains strongly correlated (Figure 6). Based on these comparisons, we hypothesize that high spike rate and synchronous firing of neighboring cells drive eye-specific segregation, even in the presence of high levels of asynchronous firing.

Since $\beta 2$-nAChRs containing nAChRs ( $\beta 2$-nAChRs) are found in both the retina and $\mathrm{dLGN}^{27}$, we cannot rule out the possibility that the altered segregation of retinogeniculate 
projections that we observe in $\beta 2-/-$ mice is due to a loss of $\beta 2$-nAChRs in dLGN neurons. A loss of $\beta 2$-nAChRs in the dLGN could influence eye-specific segregation by altering the activity of LGN neurons or their ability to undergo plasticity 9,28 . However, several lines of evidence suggest that the loss of $\beta 2$-containing nAChRs in the dLGN does not influence segregation. First, local infusion of general nAChR antagonists directly into the dLGN during the first postnatal week does not prevent eye-specific segregation ${ }^{2}$, but genetic disruption of $\beta 2$-nAChRs during this time does 3,7 . Second, retinogeniculate synapses retain the ability to undergo plasticity in $\beta 2-/-$ mice. The defect in eye-specific segregation coincides specifically with the period that retinal waves are disrupted in $\beta 2-/-$ mice. Once retinal waves are restored, during the second postnatal week, eye-specific segregation resumes ${ }^{7}$ and is complete by adulthood ${ }^{9}$. This temporal correlation suggests that it is the retinal activity disruption, which is brief, and not a central disruption, which is ongoing, that is responsible for the absence of eye-specific segregation.

Connexin 36 is also found in the mouse dLGN (see Supplemental Figure 2) and therefore may also alter the pattern of activity in dLGN neurons in addition to retinal effects. Indeed, in other areas of the brain, Cx36 is important for coordinating the activity among populations of neurons: Cx36-/- mice have decreased synchrony among neurons in the inferior olive 29 and impaired hippocampal oscillations ${ }^{30}$. However, Cx36-/- mice have normal eye-specific segregation, suggesting that the loss of Cx36 in both the retina and the dLGN is still not sufficient to influence the segregation process. Furthermore, $\beta 2-/-\mathrm{Cx} 36-/$ - double knock out mice have segregation patterns similar to $\beta 2-/$ - mice, indicating that the loss of $\mathrm{Cx} 36$ in the retina and dLGN did not have an additional effect on segregation.

Our finding that presynaptic activity patterns contain critical information for normal refinement of visual maps is consistent with recent reports. Ruthazer et al ${ }^{31}$ demonstrate a requisite role for patterned activity in sorting retinotectal axons into eye-specific regions following experimentally-induced binocular innervation of the optic tectum. They report that not all aspects of axon branch dynamics were activity-dependent -- branch extensions into sparsely innervated regions persisted in the presence of an activity blockade. We recently reported that $\beta 2-/$ - mice exhibit reduced retinotopic refinement of retinocollicular projections 14 , which is consistent with the hypothesis that patterned activity is required for refinement but not axonal elaborations ${ }^{12}$. In ferrets, intraocular injection of an immunotoxin to starburst amacrine cells significantly altered RGC firing, but eye-specific layer formation was normal ${ }^{5}$. In the immunotoxin-treated retinas, the spiking patterns of individual RGCs was altered and the correlation between neighboring RGCs was decreased. However, as in Cx36-/- mice (Figure 2, Figure 5), it is possible that even though the correlation between neighboring RGCs has decreased, the essential features for retinogeniculate segregation have been maintained. Indeed, correlated increases in intracellular calcium concentration persisted in immunotoxin-treated retinas, indicating that some amount of correlated activity was present in retinal neurons.

The finding that firing at $1 \mathrm{~Hz}$ is not correlated with eye-specific segregation places constraints on the plasticity rules that underlie refinement. Presynaptic $1 \mathrm{~Hz}$ firing robustly induces longterm synaptic depression (LTD) at many synapses ${ }^{32}$, but other firing patterns can also lead to LTD $^{33}$, including high-frequency trains or bursts (for example, see ${ }^{34}$ ). LTD has been proposed to lead to synapse withdrawal and the loss of inappropriate axonal connections in other systems 11 . Though it is not known whether retinogeniculate synapses undergo LTD, our results imply that if LTD contributes to retinogeniculate synaptic refinement, such LTD is likely to be driven by burst firing, rather than $1 \mathrm{~Hz}$ firing.

In summary, the analysis presented here highlights critical features of retinal waves for driving eye-specific segregation and provides an explanation for why not all manipulations that alter RGC firing patterns prevent segregation 5,26 or lead to desegregation 35 . 


\section{METHODS}

\section{Immunofluorescence}

Cx36 knockout mice in which the $\mathrm{Cx} 36$ coding sequence was replaced by a LacZ-IRES-PLAP reporter cassette 18 were a generous gift from David Paul at Harvard Medical School. In these mice, neurons that would normally express Cx36 now express the $\beta$-gal reporter in their cell bodies. Cx36 knockout mice were deeply anesthetized with halothane and eyes were removed and postfixed for two hours at $4^{\circ} \mathrm{C}$ in picric acid fixative. Tissue was cryoprotected in $30 \%$ sucrose with $0.1 \%$ sodium azide then cleaned and cut as an eye cup and submerged in OCT compound. $16 \mu \mathrm{m}$ frozen sections were prepared and thaw-mounted on Superfrost Plus ${ }^{\mathrm{TM}}$ microscope slides (Fisher). Sections were thoroughly washed in 1X PBS and then incubated in a solution containing $500 \mathrm{mM}$ potassium ferrocyanide, $500 \mathrm{mM}$ potassium ferricyanide, 2 $\mathrm{M} \mathrm{MgCl}_{2}, 0.3 \%$ TritonX-100, $40 \mathrm{mg} / \mathrm{ml}$ 5-bromo-4-chloro-3-indolyl- $\beta$-D-galactopyranoside (X-gal, Sigma), in $1 \mathrm{X}$ PBS (ph 7.4-7.5) at $37^{\circ} \mathrm{C}$ for approximately 30 miutes-2 hours in the dark. All tissue was dehydrated through alcohol gradients and xylene, and coverslipped with Permount. Brightfied images were acquired on an upright microscope (Zeiss Axioscope 2) with a CCD camera (Optronics).

\section{Multielectrode Recordings and Analysis}

Newborn wild type (WT) and Cx36-/- mice [postnatal day 4 (P4) -P11] were anesthetized with isofluorane and decapitated. Retinas were isolated in cold $\left(4^{\circ} \mathrm{C}\right)$ artificial cerebrospinal fluid (ACSF) containing (in mM): $119.0 \mathrm{NaCl}, 26.2 \mathrm{NaHCO}_{3}, 11$ glucose, $2.5 \mathrm{KCl}, 1.0 \mathrm{~K}_{2} \mathrm{HPO}_{4}$, $2.5 \mathrm{CaCl}_{2}$ and $1.3 \mathrm{MgCl}_{2}$. Retinas were cut into thirds and mounted, ganglion cell side up, onto filter paper.

Retina pieces were placed ganglion cell side down onto a flat, hexagonal array of 61 extracellular electrodes spaced $60 \mu \mathrm{m}$ apart from each other, with a total diameter of $480 \mu \mathrm{m}$. During recording the retina was continuously perfused with oxygenated ACSF and the bath temperature was between $32^{\circ}$ and $35^{\circ} \mathrm{C}$. The filter paper to which the retina was attached was held down with a platinum ring.

Spike times, peaks, and widths were digitized with a temporal resolution of $0.05 \mathrm{msec}^{20}$ and then stored for off-line analysis. Spikes were manually segregated into single units by selecting distinct clusters in scatter plots of spike height and width recorded on each electrode 20 and verifying the presence of a refractory period in the spike trains from each cluster. Spikes recorded on multiple electrodes were identified by temporal coincidence; only spikes from the electrode with the most clearly defined cluster were further analyzed. Using this method, between 10 and 65 unique units were identified from each retina piece. Cells were considered to be at the position of the electrode on which they were recorded. Cell-attached recordings from individual RGCs revealed spike train adaptation ${ }^{36}$, with much larger action potentials at the beginning of the train that quickly diminish in amplitude (Supplemental Figure 3). Since action potentials recorded on the multielectrode array are sorted into individual units based on their shape ${ }^{20}$, a small number of spikes may have been excluded from the analysis.

We computed several measures of the spiking properties of RGCs for each single unit recorded. Correlation index was calculated as previously described ${ }^{14,20}$. Briefly, this was calculated by determining the number of spikes from cell A that occurred within $\pm 100 \mathrm{~ms}$ of a spike in cell $\mathrm{B}$ and then dividing this by the number of spike pairs that would have occurred by random chance.

$$
\text { Correlation Index }=\frac{\mathrm{N}_{\mathrm{AB}(-0.1 \mathrm{~s},+0.1 \mathrm{~s})} \cdot \mathrm{T}}{\mathrm{N}_{\mathrm{A}(0, \mathrm{~T})} \cdot \mathrm{N}_{\mathrm{B}(0, \mathrm{~T})} \cdot(0.2 \mathrm{~s})}
$$


$\mathrm{N}_{\mathrm{AB}}=$ number of spike pairs from cells $\mathrm{A}$ and $\mathrm{B}$ for which cell $\mathrm{B}$ fires within $\pm 100 \mathrm{~ms}$ of cell A.

$\mathrm{T}=$ the total recording time.

$\mathrm{N}_{\mathrm{A}(0, \mathrm{~T})}=$ total number of spikes in cell A.

$\mathrm{N}_{\mathrm{B}(0, \mathrm{~T})}=$ total number of spikes in cell $\mathrm{B}$.

Since firing rates were often very different between both cells in a pair, the correlation index was calculated using both $\mathrm{N}_{\mathrm{AB}}$ and $\mathrm{N}_{\mathrm{BA}}$. The percentage of time cells were firing was calculated by summing the interspike intervals (ISI) less than either $1 \mathrm{~s}$ or $100 \mathrm{~ms}$ and dividing this by the total time of the recording. The average number of action potentials was calculated by summing the total number of action potentials for each 20 min recording and then dividing by the length of the recording. The median firing rate was calculated by first calculating the instantaneous firing rate for all spikes recorded for an individual unit, then determining the median for that unit. Since the distributions for each parameter measure were non-normal, averages were reported as medians and errors bars were quartiles. For statistical analysis, units from each recorded age (P4-5 and P10-11) and genotype (WT, Cx36-/-, $\beta 2-/-, \beta 2-/-C x 36-/-)$ were grouped. Within each age group, a Kruskal-Wallis test was used to compare genotypes followed by a post-hoc Dunn test to determine which genotypes were different from agematched WT. P-values less than 0.05 were taken as significant. All analysis was performed using IgorPro (Wavemetrics, Inc).

\section{Intraocular Injections and Image Analysis}

Animals were anesthetized with $3.5 \%$ isoflurane $/ 2 \% \mathrm{O}_{2}$. The eyelid was then cut open to expose the eye, and $0.1-1 \mu \mathrm{l}$ of Alexa-488 or Alexa-594 conjugated $\beta$-choleratoxin was injected using a fine glass micropipette with a picospritzer (World Precision Instruments, Sarasota, FL). The cholera toxin was allowed to transport for 24 hours, which was sufficient time for clear labeling of axons and terminals. Animals were heavily anesthetized with an overdose of isoflurane, then fixed by cardiac perfusion with $4 \%$ paraformaldehyde in PBS, and brains were sectioned coronally to $100 \mu \mathrm{m}$ on a Vibratome.

Images were analyzed as described previously ${ }^{7,22}$. Briefly, eight-bit tagged image file format images were acquired for Alexa488- or 594-labeled sections of the LGN with a CCD camera (Optronics, Goleta, CA) attached to an upright microscope (Zeiss Axioscope 2; Thornwood, NY) with a 10X objective (numerical aperture, 0.45 ). The three sections that contained the largest ipsilateral projection, corresponding to the central third of the LGN were selected, and all analysis was performed on these sections on the side of the brain with an Alexa594-labeled ipsilateral/Alexa488-labeled contralateral projection. Background fluorescence was subtracted using a rolling ball filter (NIH Image) and the grayscale was renormalized so that the range of grayscale values was from 0 to 256. IgorPro Software (Wavemetrics, Lake Oswego, OR) was used to perform two independent analyses.

First, the fraction of segregated pixels was calculated by first setting the threshold for delineating signal on the ipsilateral projection at the level at which there was no longer signal from the optic tract. Using the same images, the region around the ipsilateral projection was analyzed for segregation of ipsilateral and contralateral axons by methods described in detail in Muir-Robinson et al. (2002). Second, for each pixel, we computed the logarithm of the intensity ratio, $R=\log _{10}\left(\mathrm{~F}_{\mathrm{I}} / \mathrm{F}_{\mathrm{C}}\right)$. Where $\mathrm{F}_{\mathrm{I}}$ is the ispilateral channel fluorescence intensity and $\mathrm{F}_{\mathrm{C}}$ is the contralateral channel fluorescence intensity. We then calculated the variance of the distribution of $\mathrm{R}$ values for each section, which was used to compare the width of the 
distributions across animals. Wider distributions of $\mathrm{R}$ indicate more contra- and ipsi-dominant pixels, and therefore more segregation.

\section{Supplementary Material}

Refer to Web version on PubMed Central for supplementary material.

\section{Acknowledgements}

We thank E. J. Chichilnisky for the multielectrode array and technical support, D. Paul for the Connexin 36-/- mice, W. Pak for technical support, D. E. Feldman, N. Spitzer, and L. Boulanger for a critical reading of this manuscript. Supported in part by an NSF Graduate Research Fellowship, the Klingenstein Foundation, Whitehall Foundation, March of Dimes, McKnight Scholars Fund, and the National Institute of Health (grant no NS13528-01A1).

\section{References}

1. Shatz CJ, Stryker MP. Prenatal tetrodotoxin infusion blocks segregation of retinogeniculate afferents. Science 1988;242:87-9. [PubMed: 3175636]

2. Penn AA, Riquelme PA, Feller MB, Shatz CJ. Competition in retinogeniculate patterning driven by spontaneous activity. Science 1998;279:2108-12. [PubMed: 9516112]

3. Rossi FM, et al. Requirement of the nicotinic acetylcholine receptor beta 2 subunit for the anatomical and functional development of the visual system. Proc Natl Acad Sci U S A 2001;98:6453-8. [PubMed: 11344259]

4. Stellwagen D, Shatz CJ. An instructive role for retinal waves in the development of retinogeniculate connectivity. Neuron 2002;33:357-67. [PubMed: 11832224]

5. Huberman $\mathrm{AD}$, et al. Eye-specific retinogeniculate segregation independent of normal neuronal activity. Science 2003;300:994-8. [PubMed: 12738869]

6. Godement P, Salaun J, Imbert M. Prenatal and postnatal development of retinogeniculate and retinocollicular proejctions in the mouse. J Comp Neurol 1984;230:552-575. [PubMed: 6520251]

7. Muir-Robinson G, Hwang BJ, Feller MB. Retinogeniculate axons undergo eye-specific segregation in the absence of eye-specific layers. J Neurosci 2002;22:5259-64. [PubMed: 12097474]

8. Huberman AD, Stellwagen D, Chapman B. Decoupling eye-specific segregation from lamination in the lateral geniculate nucleus. J Neurosci 2002;22:9419-29. [PubMed: 12417667]

9. Grubb MS, Rossi FM, Changeux JP, Thompson ID. Abnormal functional organization in the dorsal lateral geniculate nucleus of mice lacking the beta 2 subunit of the nicotinic acetylcholine receptor. Neuron 2003;40:1161-72. [PubMed: 14687550]

10. Butts DA. Retinal waves: implications for synaptic learning rules during development. Neuroscientist 2002;8:243-53. [PubMed: 12061504]

11. Katz LC, Shatz CJ. Synaptic activity and the construction of cortical circuits. Science 1996;274:11338. [PubMed: 8895456]

12. Ruthazer ES, Cline HT. Insights into activity-dependent map formation from the retinotectal system: a middle-of-the-brain perspective. J Neurobiol 2004;59:134-46. [PubMed: 15007832]

13. Mooney R, Penn AA, Gallego R, Shatz CJ. Thalamic relay of spontaneous retinal activity prior to vision. Neuron 1996;17:863-74. [PubMed: 8938119]

14. McLaughlin T, Torborg CL, Feller MB, O'Leary DD. Retinotopic map refinement requires spontaneous retinal waves during a brief critical period of development. Neuron 2003;40:1147-60. [PubMed: 14687549]

15. Singer JH, Mirotznik RR, Feller MB. Potentiation of L-Type Calcium Channels Reveals Nonsynaptic Mechanisms that Correlate Spontaneous Activity in the Developing Mammalian Retina. J Neurosci 2001;21:8514-22. [PubMed: 11606640]

16. Guldenagel M, et al. Expression patterns of connexin genes in mouse retina. J Comp Neurol 2000;425:193-201. [PubMed: 10954839]

17. Sohl G, Guldenagel M, Traub O, Willecke K. Connexin expression in the retina. Brain Res Brain Res Rev 2000;32:138-45. [PubMed: 10751663] 
18. Deans MR, Gibson JR, Sellitto C, Connors BW, Paul DL. Synchronous activity of inhibitory networks in neocortex requires electrical synapses containing connexin36. Neuron 2001;31:477-85. [PubMed: $11516403]$

19. Deans MR, Volgyi B, Goodenough DA, Bloomfield SA, Paul DL. Connexin36 is essential for transmission of rod-mediated visual signals in the mammalian retina. Neuron 2002;36:703-12. [PubMed: 12441058]

20. Meister M, Pine J, Baylor DA. Multi-neuronal signals from the retina: acquisition and analysis. J Neurosci Methods 1994;51:95-106. [PubMed: 8189755]

21. Demas J, Eglen SJ, Wong RO. Developmental loss of synchronous spontaneous activity in the mouse retina is independent of visual experience. J Neurosci 2003;23:2851-60. [PubMed: 12684472]

22. Torborg CL, Feller MB. Unbiased Analysis of Bulk Axonal Segregation Patterns. Journal of Neuroscience Methods 2004; 135

23. Bansal A, Singer JH, Hwang B, Feller MB. Mice lacking specific nAChR subunits exhibit dramatically altered spontaneous activity patterns and reveal a limited role for retinal waves in forming ON/OFF circuits in the inner retina. J Neurosci 2000;20:7672-81. [PubMed: 11027228]

24. Meister M, Wong RO, Baylor DA, Shatz CJ. Synchronous bursts of action potentials in ganglion cells of the developing mammalian retina. Science 1991;252:939-43. [PubMed: 2035024]

25. Wong RO, Meister M, Shatz CJ. Transient period of correlated bursting activity during development of the mammalian retina. Neuron 1993;11:923-38. [PubMed: 8240814]

26. Stellwagen D, Shatz CJ, Feller MB. Dynamics of retinal waves are controlled by cyclic AMP. Neuron 1999;24:673-85. [PubMed: 10595518]

27. Zoli M, Lena C, Picciotto MR, Changeux JP. Identification of four classes of brain nicotinic receptors using beta2 mutant mice. J Neurosci 1998;18:4461-72. [PubMed: 9614223]

28. Grubb MS, Thompson ID. Visual response properties in the dorsal lateral geniculate nucleus of mice lacking the beta2 subunit of the nicotinic acetylcholine receptor. J Neurosci 2004;24:8459-69. [PubMed: 15456819]

29. Long MA, Deans MR, Paul DL, Connors BW. Rhythmicity without synchrony in the electrically uncoupled inferior olive. J Neurosci 2002;22:10898-905. [PubMed: 12486184]

30. Buhl DL, Harris KD, Hormuzdi SG, Monyer H, Buzsaki G. Selective impairment of hippocampal gamma oscillations in connexin-36 knock-out mouse in vivo. J Neurosci 2003;23:1013-8. [PubMed: 12574431]

31. Ruthazer ES, Akerman CJ, Cline HT. Control of axon branch dynamics by correlated activity in vivo. Science 2003;301:66-70. [PubMed: 12843386]

32. Bear MF. A synaptic basis for memory storage in the cerebral cortex. Proc Natl Acad Sci U S A 1996;93:13453-9. [PubMed: 8942956]

33. Bi G, Poo M. Synaptic modification by correlated activity: Hebb's postulate revisited. Annu Rev Neurosci 2001;24:139-66. [PubMed: 11283308]

34. Birtoli B, Ulrich D. Firing mode-dependent synaptic plasticity in rat neocortical pyramidal neurons. J Neurosci 2004;24:4935-40. [PubMed: 15163685]

35. Chapman B. Necessity for Afferent Activity to Maintain Eye-Specific Segregation in Ferret Lateral Geniculate Nucleus. Science 2000;287:2479-2482. [PubMed: 10741966]

36. Wang GY, Ratto G, Bisti S, Chalupa LM. Functional development of intrinsic properties in ganglion cells of the mammalian retina. J Neurophysiol 1997;78:2895-903. [PubMed: 9405510] 

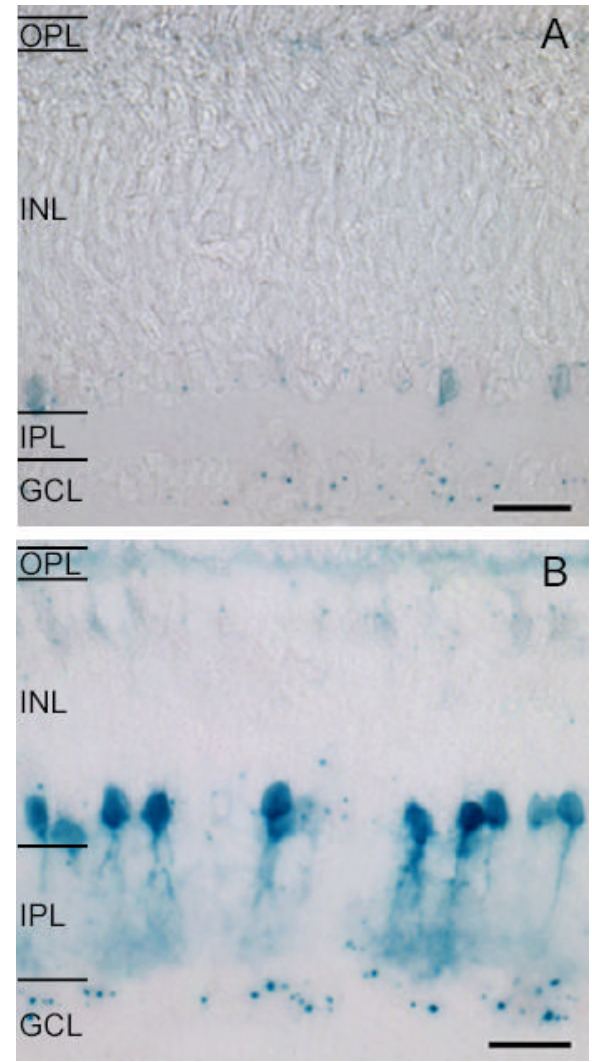

Figure 1. $\beta$-galactosidase expression in the inner retina increases during postnatal development A. P4 $\beta$-gal reaction product is localized as discrete puncta in the GCL and in cell bodies in the proximal INL. Faint labeling of the OPL is present.

B. P10 $\beta$-gal reaction product reveals an increase in the number of somata in the proximal and distal INL and in the GCL. Scale $=20 \mu \mathrm{m}$.

OPL = outer plexiform layer, INL = inner nuclear layer, IPL = inner plexiform layer, GCL = ganglion cell layer. 


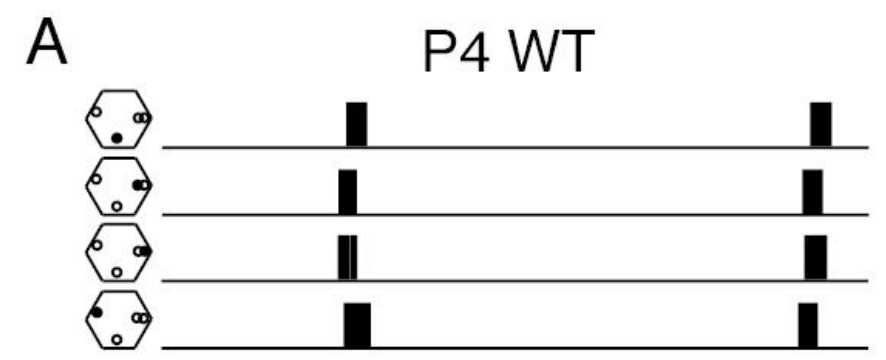

B
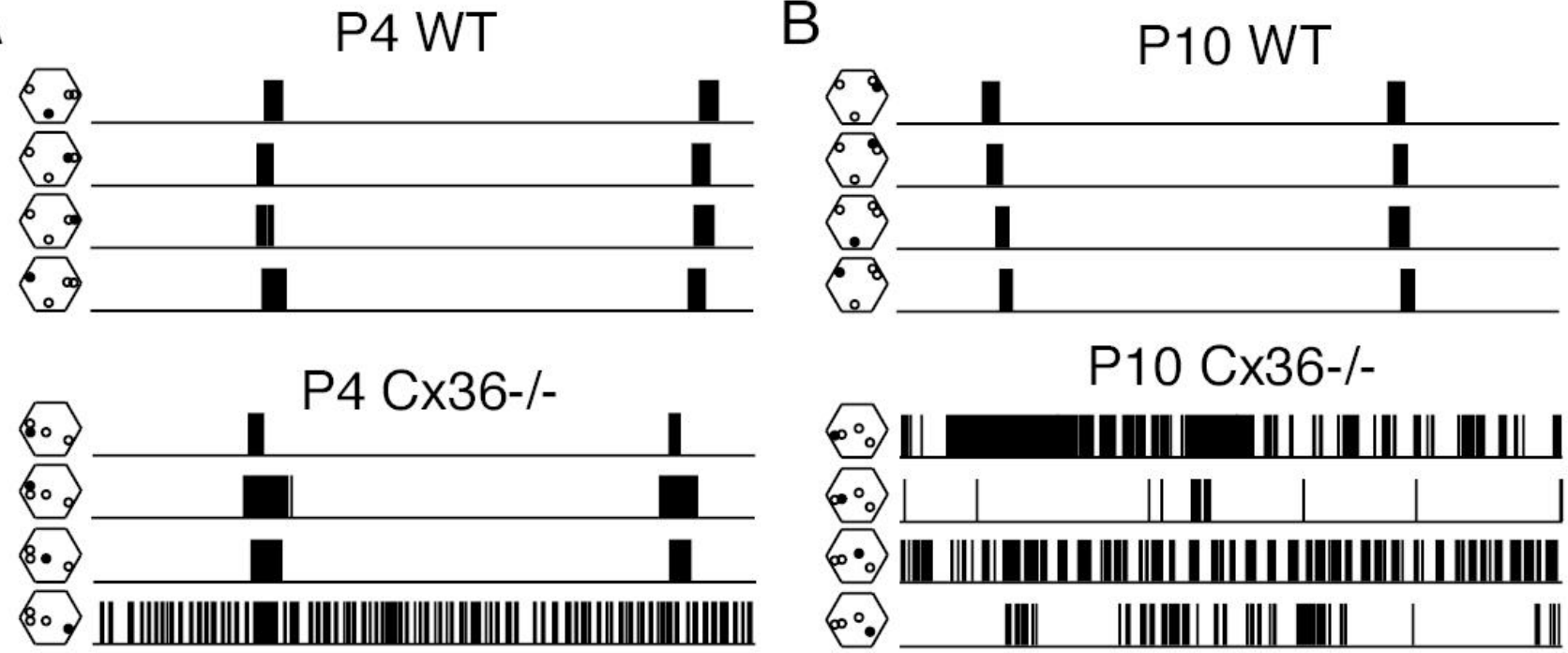

P10 Cx36-/-

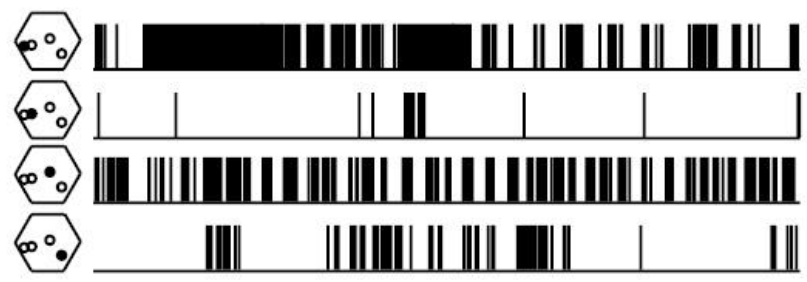

$15 \mathrm{sec}$

Figure 2. Spike patterns are disrupted in Cx36-/- retinas

A. Spike trains recorded simultaneously with a multielectrode array (MEA) from four representative neurons in WT (left) and Cx36-/- (right) retinas at P4.

B. Spike trains from four RGCs in WT (left) and Cx36-/- (right) retinas at P10. Hexagons to the left of each spike train show the position (filled circle) of the electrode on the multielectrode array that recorded spikes relative to other represented units (open circles). The maximum extent of the array is $480 \mu \mathrm{m}$. 

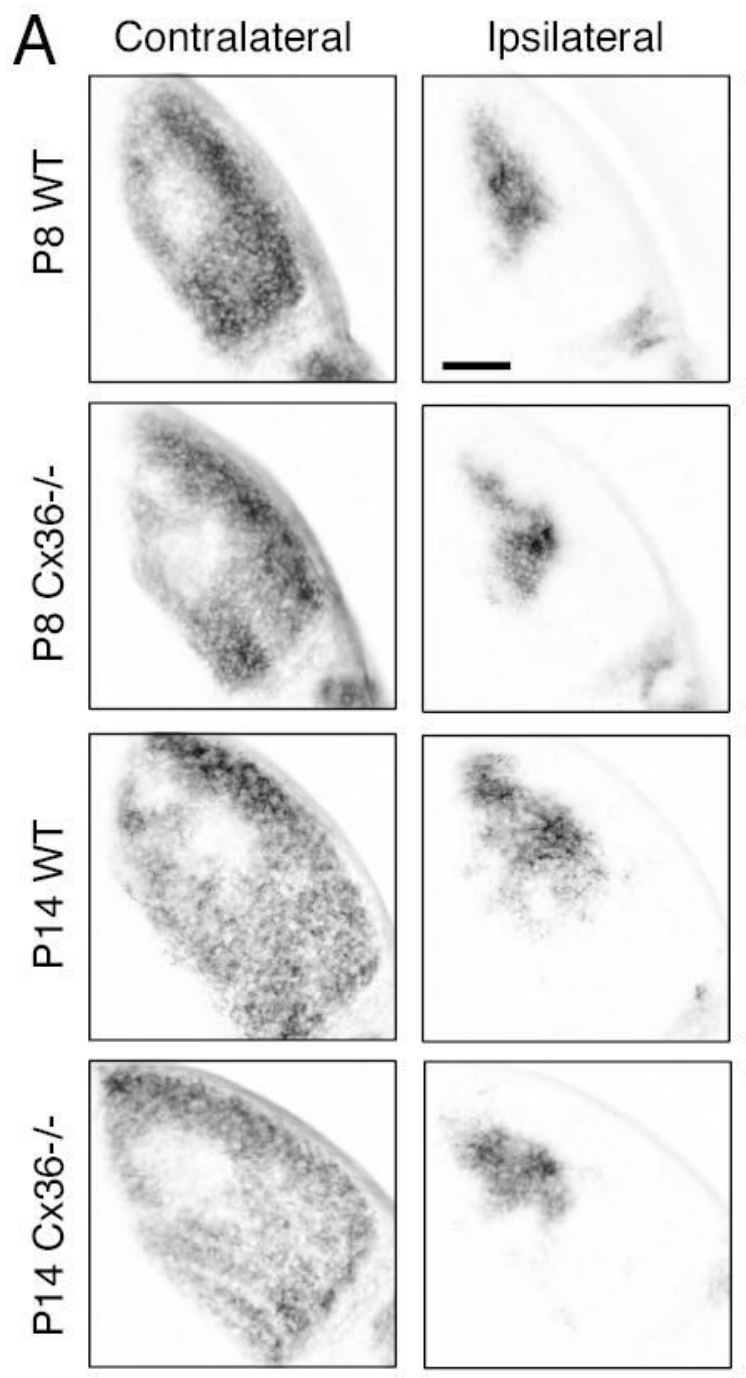

Ipsilateral
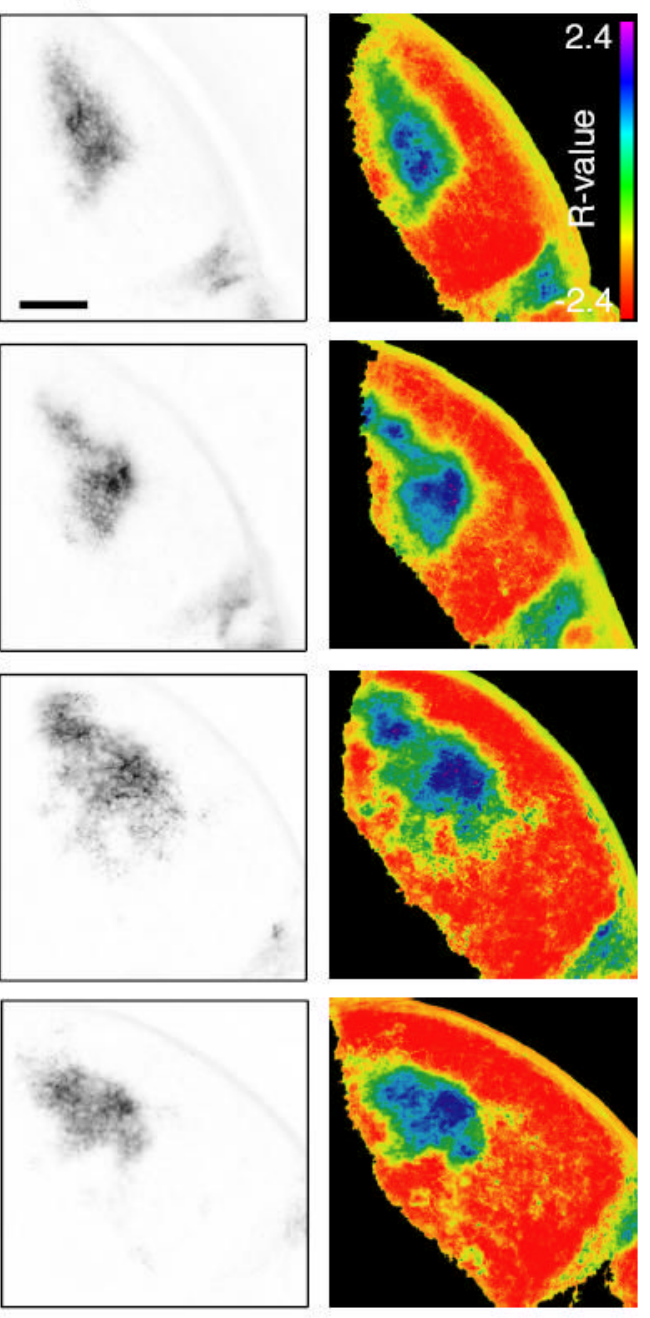

B
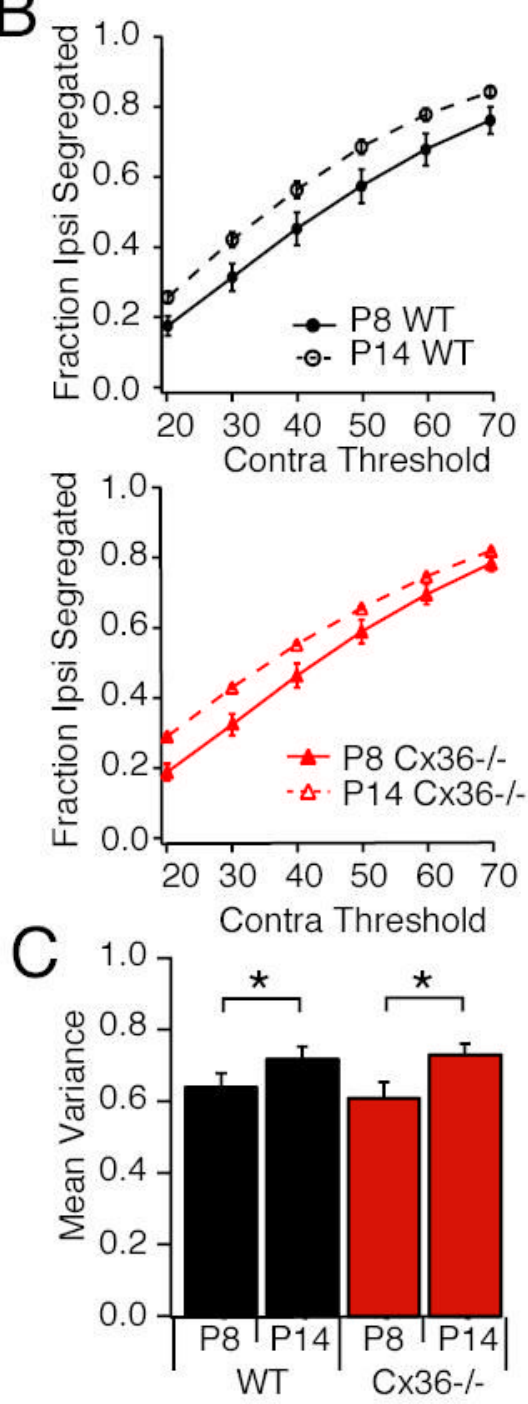

Figure 3. Cx36-/- mouse dLGN at P8 and P14 have normal segregation of ipsilateral and contralateral axon terminals

A. Fluorescence images of contralateral (column 1) and ipsilateral (column 2) projections in the same LGN slice. Column 3: Images of the dLGN pseudocolored according to the R-value for each pixel where $\mathrm{R}=\log _{10}\left(\mathrm{~F}_{\mathrm{I}} / \mathrm{F}_{\mathrm{C}}\right)$. Scale bar $=200 \mu \mathrm{m}$.

B. Summary of the fraction of pixels with ipsilateral signal alone as a function of contralateral noise threshold. N=5 P8 WT, P14 WT and P14 Cx36-/- mice, N=9 P8 Cx36-/- mice,3 sections/ mouse. Error bars = standard error of the mean. Asterisks reflect significance $(\mathrm{p}<0.05) \mathrm{C}$. Summary of the variance of the R distributions for the same images analyzed in part B. Error bars $=$ standard error of the mean. 


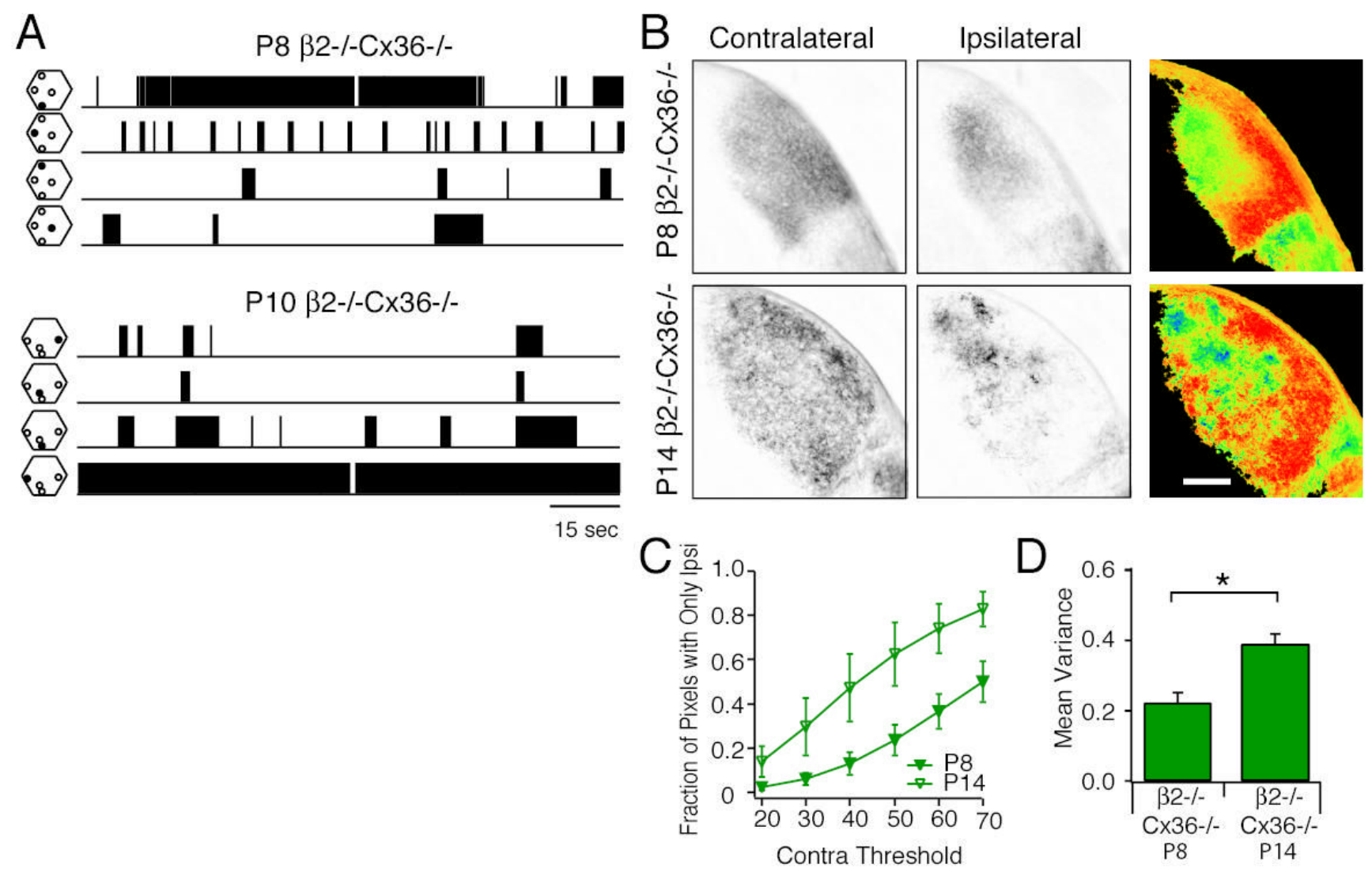

Figure 4. $32-/-\mathrm{Cx} 36-/-$ mice have disrupted spike trains and local segregation of ipsilateral and contralateral axon terminals in the absence of layers

A. Spike trains of four different neurons from a P8 $\beta 2-/-C x 36-/-$ retina (top), and a P10 $\beta 2-/-$ Cx36-/- retina (bottom). Hexagons to the left of each spike train show the position (filled circle) of the electrode on the multielectrode array that recorded spikes.

B. Fluorescence images of contralateral (left column) and ipsilateral (center column) projections and pseudocolor representation according to the $\mathrm{R}$ value for each pixel (third column) in the same LGN slice. Scale bar $=200 \mu \mathrm{m}$.

C. Summary of the fraction of pixels with ipsilateral signal alone as a function of contralateral threshold. Error bars = standard error of the mean. Asterisks reflect significance $(\mathrm{p}<0.05)$ D. Summary of the variance of the R-distributions. Error bars = standard error of the mean. $\mathrm{N}=5 \mathrm{P} 8$ and P14 $32-/-\mathrm{Cx} 36-/-$ mice, 3 sections/mouse. Asterisks reflect significance $(\mathrm{p}<0.05)$ 

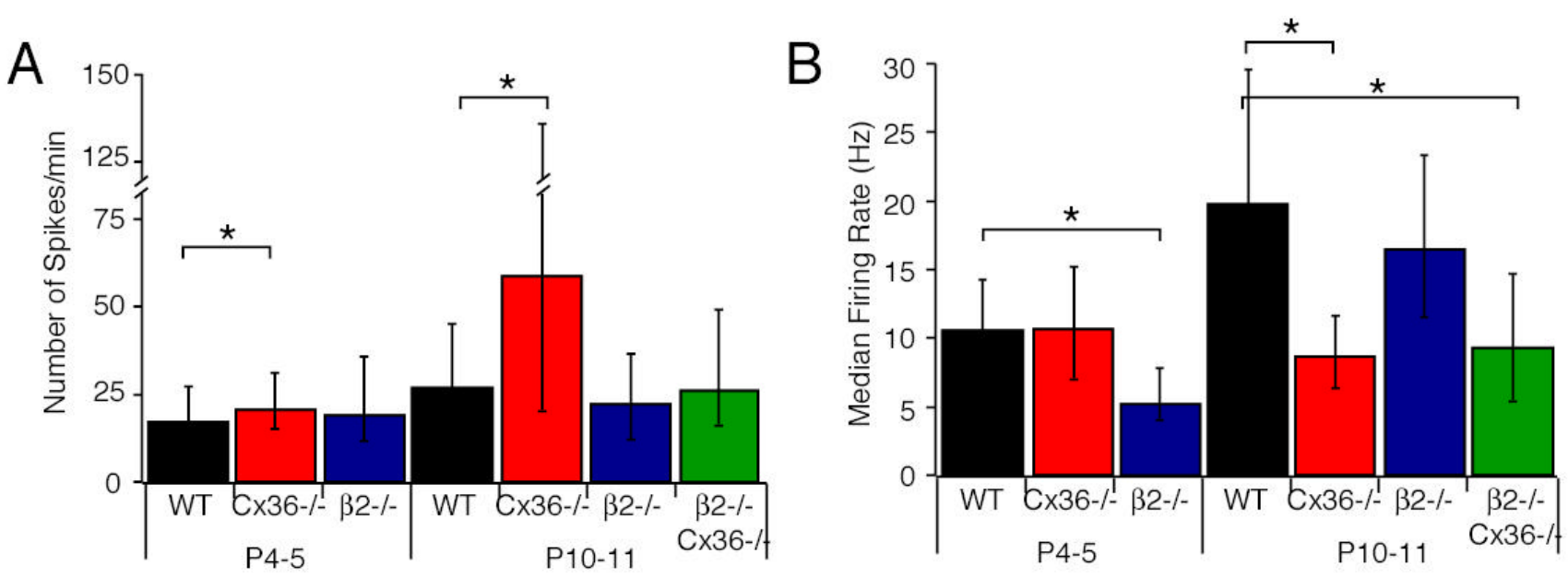

C

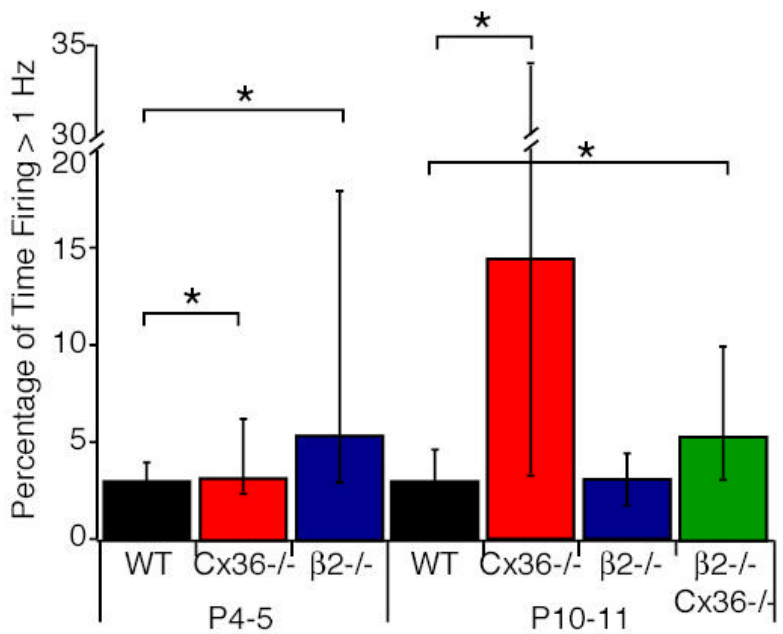

$D$

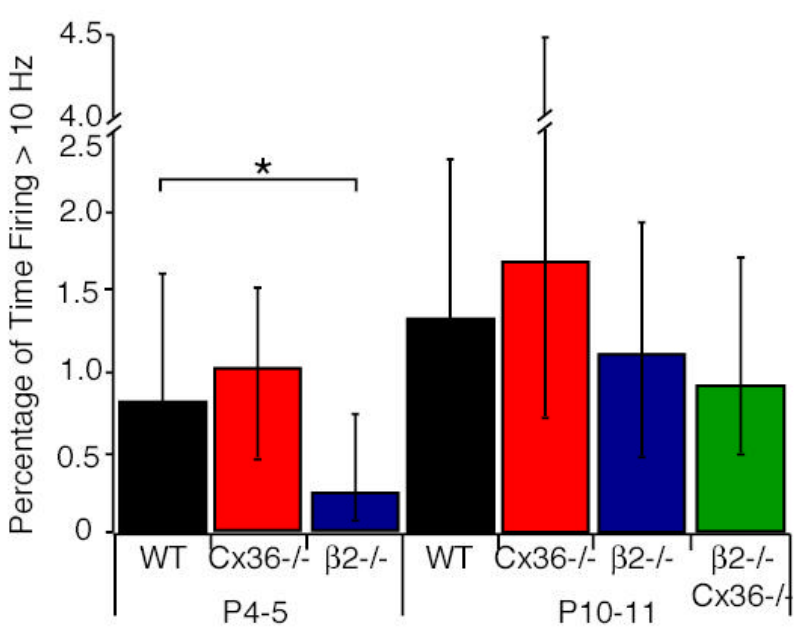

Figure 5. Individual cell firing patterns contain features that differentiate WT, Cx36-/- and ß2-/and $\beta 2-/-\mathrm{Cx} 36-/$ - mice

Summary of the total number of recorded spikes normalized by the length of the recording (A), the median instantanous firing rates in each cell (B), the percentage of time that RGCs fired at greater than $1 \mathrm{~Hz}(\mathrm{C})$, and the percentage of time that RGCs fired at greater than $10 \mathrm{~Hz}(\mathrm{D})$. For A, B, C, and D the numbers of cells analyzed were $\mathrm{n}=75$ cells, 5 retinas $(\mathrm{P} 4-5 \mathrm{WT}) ; \mathrm{n}=115$ cells, 6 retinas (P10-11 WT), $\mathrm{n}=74$ cells, 5 retinas (P4-5 Cx36-/), $\mathrm{n}=199$ cells, 6 retinas (P10-11 $\mathrm{Cx} 36-/-) ; \mathrm{n}=91$ cells, 4 retinas (P4-5 $\beta 2-/-)$; $\mathrm{n}=87,6$ retinas $(\mathrm{P} 10-11 \beta 2-/-)$; $\mathrm{n}=121$ cells, 6 retinas (P10-11 $\beta 2-/-C x 36-/-)$. Since most distributions were non-normal, all averages are reported as medians, and error bars are quartiles. Asterisks reflect significance $(\mathrm{p}<0.05)$. 

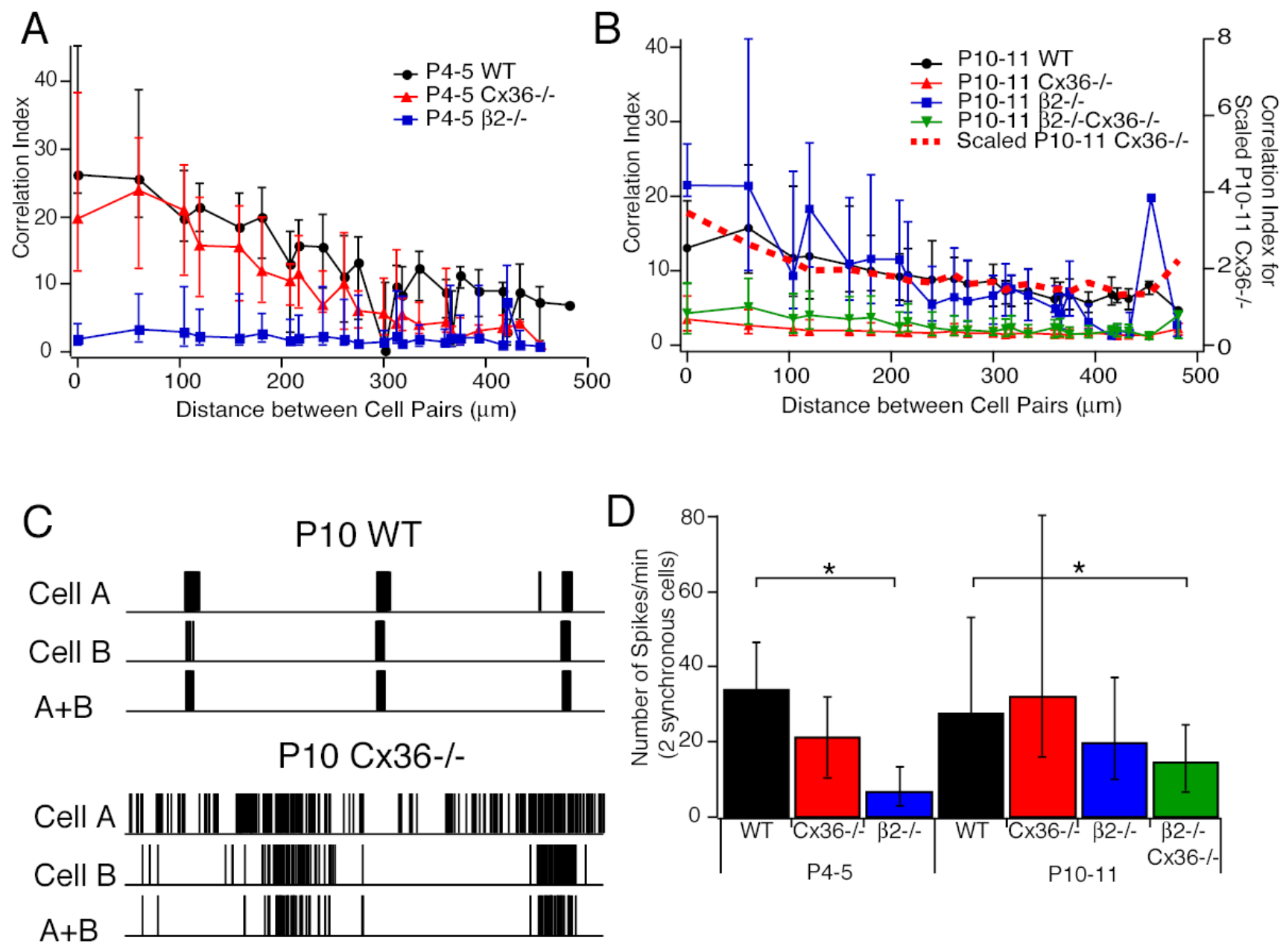

P4 $\beta 2-/-$

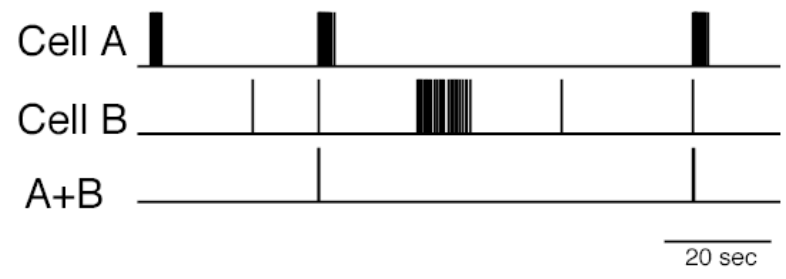

Figure 6. Correlation index is decreased in both $\mathrm{P4-5} \beta 2$-/- retinas and P10-11 Cx36-/- retinas. A. Correlation index computed for pairs of spike trains and plotted as a function of the distance between the cells for $\mathrm{P} 4-5$ retinas. $\mathrm{n}=1012$ pairs, 5 retinas $(\mathrm{P} 4-5 \mathrm{WT}) ; \mathrm{n}=1112$ pairs, 5 retinas (P4-5 Cx36-/); $\mathrm{n}=2224$ pairs, 4 retinas (P4-5 $\beta 2-/-)$.

B. Correlation index computed for pairs of spike trains and plotted as a function of the distance between the cells for P10-11 retinas. The dotted line was obtained by scaling the P10-11 Cx36-/- mouse correlation index by a factor of 4 . $n=2364$ pairs, 6 retinas (P10-11 WT); $n=7724$ pairs, 6 retinas (P10-11 Cx36-/-); $\mathrm{n}=1328$ pairs, 6 retinas (P10-11 $\beta 2-/-)$; $\mathrm{n}=3618$ pairs, 6 retinas (P10-11 $32-/-C x 36-/-)$.

$\mathrm{C}$. The spike trains from 2 neighboring cells (cell A and cell B) are used to generate a third spike train $(A+B)$. This third spike train includes those spikes that occurred within $\pm 100 \mathrm{~ms}$ 
of a spike from the other cell. Examples are shown for P10 WT(top), P10 Cx36-/- (middle), and P4 $\beta 2-/-$ (bottom).

D. Summary of the total number of spikes that are synchronous within \pm 100 ms between 2 cells that were recorded on the same or neighboring electrodes. Since some distributions were non-normal, all averages are reported as medians, and error bars are quartiles. Asterisks reflect significance $(\mathrm{p}<0.05) . \mathrm{n}=51$ pairs, 5 retinas $(\mathrm{P} 4-5 \mathrm{WT}) ; \mathrm{n}=103$ pairs, 6 retinas $(\mathrm{P} 10-11 \mathrm{WT})$, $\mathrm{n}=66$ pairs, 5 retinas $(\mathrm{P} 4-5 \mathrm{Cx} 36-/), \mathrm{n}=347$ pairs, 6 retinas $(\mathrm{P} 10-11 \mathrm{Cx} 36-/-)$; $\mathrm{n}=144$ pairs, 4 retinas (P4-5 $\beta 2-/-)$; $\mathrm{n}=56$ pairs, 6 retinas (P10-11 $\beta 2-/-)$; $=190$ pairs, 6 retinas (P10-11 $\beta 2-/-$ Cx36-/-). 
A

Control

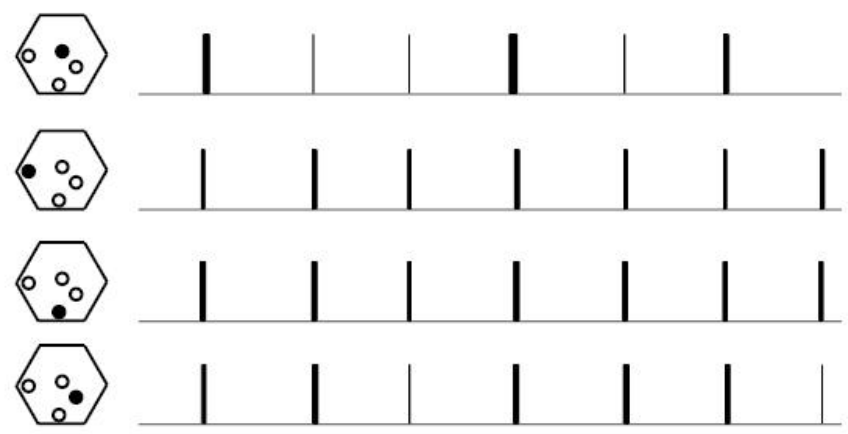

B

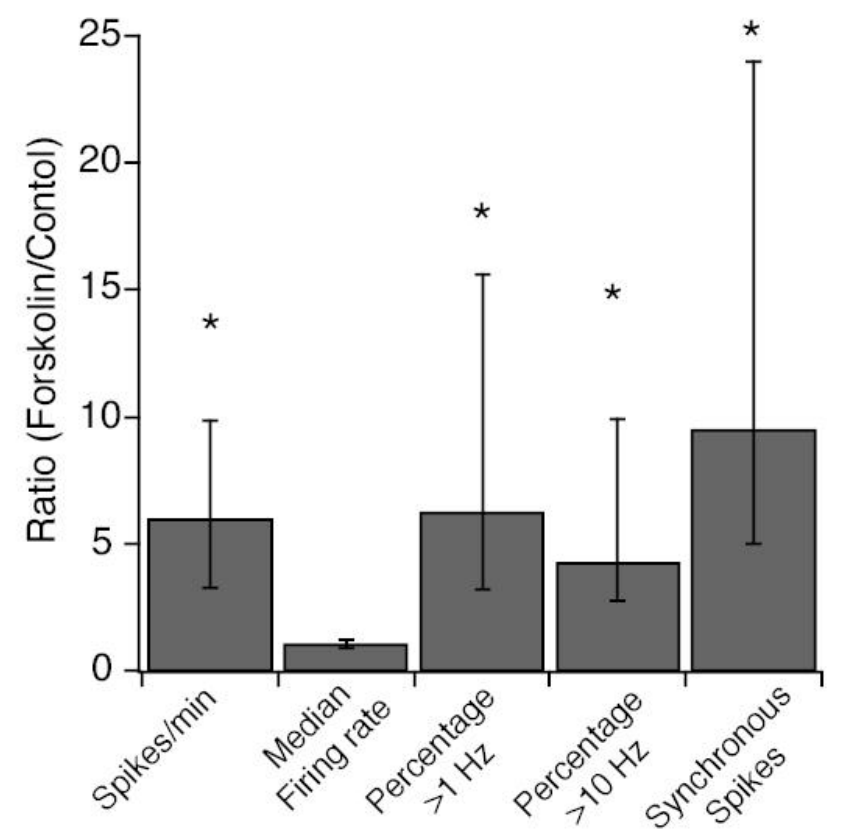

$2 \mu \mathrm{M}$ Forskolin

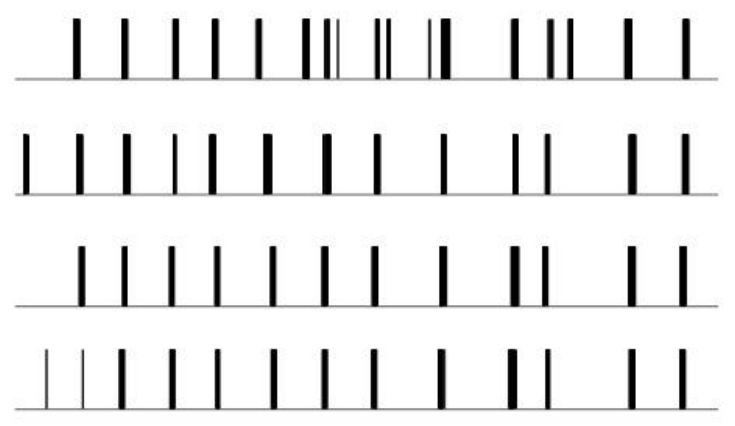

$1 \mathrm{~min}$

Figure 7. Bath application of adenylate cyclase activators enhances retinal firing but maintains appropriate features for driving eye-specific segregation A. Spikes trains of 4 different neurons from a P4-5 WT retina in the absence (left) and presence (right) of $2 \mu \mathrm{M}$ forskolin. Hexagons to the left of each spike train show the position (filled circle) of the electrode on the multielectrode array that recorded spikes. Total length of control and drug application recordings is 20 minutes.

B. Summary of effects of forskolin $(2 \mu \mathrm{M})$ on the spike train parameters defined in Figures 5 and 6. Data is presented as a ratio of the value of a parameter in the presence of forskolin normalized to its value in control solutions. This ratio was computed for each cell in the population; data was respresented as medians and quartiles. $n=24$ cells, 2 retinas for first 4 columns and $n=25$ pairs, 2 retina for the number of syncrhonized spikes. Significance was assayed by a Wilcoxon signed rank test comparing control to forskolin for each parameter. 